\title{
COMPARAÇÃO DAS BOAS PRÁTICAS ENTRE RESTAURANTES COMERCIAIS DA REGIÃO CENTRAL E REGIÃO SUL DA CIDADE DE SÃO PAULO (SP)
}

\section{GOOD PRATICES IN RESTAURANTS FROM CENTRAL AND SOUTHERN REGIONS OF THE CITY OF SÃO PAULO}

\author{
Mônica Glória Neumann Spinelli' \\ Jaqueline Maria Coelho ${ }^{2}$ \\ Ana Lúcia de Freitas Saccol ${ }^{3}$
}

RESUMO: A expressão "Boas Práticas" (BP) é utilizada para indicar um conjunto de ações aplicadas à produção de alimentos, com a finalidade de assegurar a qualidade dos produtos e prevenir riscos relacionados à saúde do consumidor. Este estudo teve por objetivo avaliar e comparar as Boas Práticas em restaurantes comerciais das regiões central e sul do município de São Paulo (SP). Aplicou-se um check list para o diagnóstico das BP e cálculo dos percentuais de adequação geral e por grupos de itens em 32 estabelecimentos, dezesseis de cada região. Foram classificados como grupo 1 os itens que apresentaram de 76 a 100\% de conformidade com as BP, grupo 2 os que tiveram de 51 a $75 \%$ e grupo 3 de 0 a 50\%. Verificou-se que a maioria dos restaurantes da região central foram classificados como grupo 2 e os requisitos com maior conformidade foram os relacionados ao controle integrado de vetores e pragas urbanas (85\%) e abastecimento de água (82,9\%). A maioria dos restaurantes da região sul foram classificados como grupo 1 e os requisitos com maior conformidade foram os relacionados ao abastecimento de água (89,3\%) e exposição ao consumo do alimento preparado (88,4\%). Pode-se concluir que, de um modo geral, os restaurantes das regiões avaliadas demonstraram boa adequação em relação às $B P$, porém a região sul apresentou melhores resultados que os da região central e que os restaurantes que apresentaram custo mais elevado das refeições tiveram melhor qualidade dos serviços prestados.

Palavras-chave: manipulação de alimentos; boas práticas de manipulação; vigilância sanitária; alimentação coletiva; segurança alimentar e nutricional.

ABSTRACT: Good Practices (GPs) are used to indicate a set of actions applied to food production, which ensure the quality of products and prevent risks to consumer health. The present study evaluated and compared the good practices in 32 restaurants in the central and southern districts of São Paulo (SP), sixteen restaurants in each region. A checklist was applied to diagnosis the GPs and calculate the percent of adequacy. We classified as a group 1 those items between 76 and 100\% of adequacy of GPs, group 2 had between 51 and 75\%, and group 3 were those with less than 50 . Most of the restaurants in the central region were classified as group 2 with best adherence rates were related to the integrated control of vectors and urban pests (85\%) and water supply (82.9\%). Most of the restaurants in the southern district were classified as group 1 with best adherence rates related to water supply (89.3\%) and finished product served to the consumer (88.4\%). The restaurants of the assessed regions showed good adequacy in relation to GPs. However, the southern region yielded better results than those of the central region, and more expensive restaurants had better quality of presented services.

Keywords: food handling; good manipulation practices; health surveillance; collective feeding; food security.

\footnotetext{
${ }^{1}$ Doutora em Saúde Pública - Universidade de São Paulo - USP e Professora Adjunta da Universidade Presbiteriana Mackenzie - UPM. E-mail: spinelli@mackenzie.br.

${ }^{2}$ Graduada em Nutrição - UPM. E-mail: jacq.coelho@hotmail.com.

${ }^{3}$ Doutora em Ciência e Tecnologia de Alimentos - Universidade Federal de Santa Maria - UFSM e Docente do Centro Universitário Franciscano - Unifra. E-mail: alsaccol@yahoo.com.br.
} 


\section{INTRODUÇÃO}

No Brasil, estima-se que, de cada cinco refeições, uma é feita fora de casa; na Europa, duas em cada seis; e, nos EUA, uma em cada duas. Esses números indicam que ainda pode haver um grande aumento e desenvolvimento dos estabelecimentos que produzem alimentos para consumo imediato no país (ARAÚJO, 2002).

O Comitê da Organização Mundial da Saúde e a Organização das Nações Unidas para Alimentação e Agricultura (WHO/FAO) admitem que doenças oriundas de alimentos contaminados seja, provavelmente, o maior problema de saúde no mundo contemporâneo. Os principais problemas são consequências do reaquecimento e refrigeração inadequados e da preparação de alimentos com muita antecedência, aumentando o tempo de espera (WEINGOLD; GUZEWICH; FUDALA, 1994).

Foram notificados à Secretaria de Vigilância em Saúde do Ministério da Saúde, entre 1999 e 2009, 6.672 surtos causados por alimentos contaminados, com o acometimento de 128.954 pessoas e registro de 82 óbitos, sendo que as regiões Sul e Sudeste foram as que mais notificaram, uma vez que a notificação está diretamente relacionada ao conhecimento da população para comunicar o evento, à sensibilidade dos profissionais de saúde e, principalmente, à implantação dessa vigilância nos municípios (BRASIL, 2009).

Segundo Braite de Oliveira (2013), de 2008 a 2010, foram notificados 1.831 surtos de DTA no estado de São Paulo, variando de 374 em 2008 a 863 em 2010, com um total de 33.534 casos, nove óbitos e letalidade de $0,02 \%$.

A necessidade de aperfeiçoamento das ações de controle sanitário na área de alimentos levou o Ministério da Saúde (MS) e a Agência Nacional de Vigilância Sanitária (ANVISA) (BRASIL, 1993, 1997, 2002, 2004) a elaborarem portarias que estabelecessem orientações necessárias para inspeção sanitária, por meio da verificação do sistema APPCC da empresa produtora e de serviços de alimentos e os aspectos que devem ser levados em conta para a aplicação das BP.

As Boas Práticas (BPs) são normas de procedimentos a fim de atingir um determinado padrão de identidade e qualidade de um produto e/ou serviço na área de alimentos, incluindo-se bebidas, utensílios e materiais em contato com alimentos (ANVISA, 2005), visando à promoção e à certificação da qualidade e da segurança do alimento (TOMICH et al., 2005). Seus princípios são pré-requisitos para implantação do sistema de análise de perigos e pontos críticos de controle (APPCC), no qual ocorre o controle de cada etapa do processamento. Atua visando à redução de riscos de ocorrência de microorganismos através do controle dos procedimentos em pontos críticos específicos durante a produção de alimentos (CASTRO; SCHIMIDT; LEITÃO, 2002).

Melhorias nos métodos de processamento dos alimentos, como a adoção e aplicação de programas de BPs, e a educação dos responsáveis pelo fornecimento de alimentos, com certeza reduziriam a incidência das doenças transmitidas por alimentos. É essencial que se conheçam as causas das doenças, agentes bacterianos, locais onde os incidentes ocorrem e os fatores que contribuem para os incidentes. Muitas práticas inadequadas que ocorrem durante o processamento permitem as contaminações, a sobrevivência e a multiplicação de microrganismos patogênicos. Um 
estudo desses fatores fornece dados que podem ser utilizados na educação das pessoas envolvidas na produção de alimentos (HOBBS; ROBERTS, 1999; FORSYTHE, 2000).

De acordo com o Codex Alimentarius (2001), é direito do consumidor adquirir alimentos seguros ao consumo. A garantia da qualidade e da segurança na alimentação é direito dos consumidores em todo o mundo. Por isso, cada vez mais, as organizações públicas e as empresas do setor de alimentos têm buscado assegurar a qualidade de seus produtos e serviços.

Diante do exposto, este trabalho tem o objetivo de avaliar e comparar as Boas Práticas em restaurantes comerciais das regiões central e sul do município de São Paulo (SP).

\section{METODOLOGIA}

Foram pesquisados 32 restaurantes do tipo self service com distribuição de alimentos de forma centralizada, vendidos por quilograma e que continham informações sobre o preço das refeições - dezesseis da região central e dezesseis da região sul de São Paulo (SP).

Somente participaram da pesquisa os estabelecimentos que tinham no mínimo seis meses de funcionamento e que assinaram o Termo de Aceite em Participação na Pesquisa.

O instrumento utilizado para avaliar as condições de Boas Práticas foi um check list, desenvolvido com base na resolução RDC no. 216, de 15 de setembro de 2004, por Saccol et al. (2006) para o diagnóstico das Boas Práticas e cálculo dos percentuais de adequação geral e por grupos de itens e os resultados foram tabulados em planilha eletrônica do Microsoft Excell 97-2003.

Foram classificados como grupo 1 os itens que apresentaram de 76 a $100 \%$ de conformidades com as Boas Práticas, grupo 2 os que tiveram de 51 a $75 \%$, e grupo 3 de 0 a $50 \%$ (AVEGLIANO et al, 2010).

Pelas características da amostra, aplicou-se o Teste Exato de Fisher para análise da significância dos resultados.

O presente estudo foi aprovado em seus aspectos éticos e metodológicos pelo CEP 0108.0.243.000-10.

\section{RESULTADOS E DISCUSSÃO}

Realizou-se a análise dos grupos de adequação entre os restaurantes das duas regiões, como pode ser verificado na Tabela 1. Nessa Tabela, também pode ser observado que os restaurantes da região sul apresentaram melhor desempenho em relação às conformidades com as Boas Práticas, quando comparados aos da região central. 
Tabela 1 - Classificação dos restaurantes self service da região central e da região sul da cidade de São Paulo (SP) segundo percentual de adequação. São Paulo, 2012

\begin{tabular}{lcc}
\hline \multicolumn{1}{c}{ Grupo de Adequação (\%) } & Região Central (\%) & Região Sul (\%) \\
\hline Grupo 1 - 76 a 100 & 31,2 & 50,0 \\
Grupo 2 - 51 a 75 & 56,2 & 43,7 \\
Grupo 3 - 0 a 50 & 12,5 & 6,3 \\
\hline
\end{tabular}

\section{Tabela 2 - Percentual de adequação por grupo de itens dos restaurantes self service da} região central e região sul de São Paulo (SP). São Paulo, 2012

\begin{tabular}{lcccccc}
\hline \multirow{2}{*}{ Grupo de itens } & & \multicolumn{2}{c}{$\begin{array}{c}\text { Adequação região } \\
\text { central }\end{array}$} & \multicolumn{2}{c}{$\begin{array}{c}\text { Adequação } \\
\text { região sul }\end{array}$} & \\
\cline { 3 - 7 } & & $\mathbf{n}$ & $\%$ & $\mathbf{n}$ & $\%$ & Valor p \\
\hline Edificação, Instalações, & sim & 42 & 76,7 & 44 & 80,5 & 0,818 \\
Equipamentos, Móveis e & não & 13 & 23,3 & 11 & 19,5 & \\
Utensílios & sim & 12 & 70,8 & 15 & 86,2 & 0,398 \\
Higienização Instalações, & não & 5 & 29,2 & 2 & 13,8 & \\
Equipamentos, Móveis e & sim & 5 & 85,0 & 5 & 84,3 & 1,000 \\
Utensílios & não & 1 & 15,0 & 1 & 15,7 & \\
Controle Integrado de & sim & 7 & 82,9 & 7 & 89,3 & 1,000 \\
Vetores e Pragas Urbanas & não & 1 & 17,1 & 1 & 10,7 & \\
Abastecimento de Água & sim & 2 & 60,4 & 3 & 78,1 & 1,000 \\
& não & 2 & 39,6 & 1 & 21,9 & \\
Manejo dos Resíduos & sim & 9 & 71,0 & 11 & 84,9 & 0,645 \\
Manipuladores & não & 4 & 29,0 & 2 & 15,1 & \\
Matérias-primas, Ingredientes & sim & 9 & 70,6 & 10 & 76,1 & 1,000 \\
e Embalagens & não & 4 & 29,4 & 3 & 23,9 & \\
Preparação do Alimento & sim & 16 & 67,8 & 20 & 82,3 & 0,318 \\
& não & 8 & 32,2 & 4 & 17,7 & \\
Armazenamento e Transporte & sim & 3 & 42,2 & 5 & 75,0 & 0,545 \\
do Alimento Preparado & não & 3 & 57,8 & 1 & 25,0 & \\
Exposição ao Consumo do & sim & 1 & 11,4 & 9 & 88,4 & 0,001 \\
Alimento Preparado & não & 9 & 88,6 & 1 & 11,6 & \\
Documentação e Registro & sim & 7 & 28,0 & 7 & 30,3 & 1,000 \\
& não & 17 & 72,0 & 17 & 69,7 & \\
\hline
\end{tabular}

Observou-se (Tabela 1) que os restaurantes avaliados neste estudo obtiveram um desempenho melhor que os da pesquisa realizada por Akutsu et al. (2005) no Distrito Federal, que mostrou que $67 \%$ dos restaurantes comerciais avaliados foram classificados no grupo 3 (entre 0 e $50 \%$ de conformidades com as Boas Práticas), embora esse resultado ainda deixe a desejar, se considerado o fato de que apenas $31,2 \%$ e $50 \%$ dos restaurantes da zona Central e da zona Sul, respectivamente, foram classificados como grupo 1.

Comparados os percentuais de adequação, por grupo de itens dos restaurantes das duas 
regiões, observou-se um percentual estatisticamente significativo favorável à região Sul apenas em relação à exposição ao alimento preparado. Assim como no estudo de Saccol et al. (2013), as maiores adequações foram relativas ao Controle Integrado de Vetores e Pragas Urbanas e ao Abastecimento de Água.

Conforme observado na Tabela 2, constatou-se que o percentual médio de adequação dos restaurantes da região sul foram bastante diferenciados em relação aos da região central. Os requisitos com maior conformidade de adequação com as Boas Práticas nos restaurantes da região central foram os relacionados ao controle integrado de vetores e pragas urbanas $(85 \%)$ e ao abastecimento de água (82,9\%). Já, os restaurantes da região sul apresentaram como requisitos com maior conformidade os relacionados ao abastecimento de água $(89,3 \%)$ e à exposição ao consumo de alimento preparado $(88,4 \%)$.

Segundo Mürmann, Mallmann e Dilkin (2007), as áreas de armazenamento e manipulação de alimentos são muito atrativas para a infestação de pragas, uma vez que fornecem os três principais subsídios de sobrevivência (alimento, abrigo e água). Devido à importância dos insetos e roedores como transmissores de doenças, os locais onde se elaboram, fracionam, acondicionam, depositam ou armazenam alimentos não embalados, devem apresentar dispositivos que impeçam a atração, o abrigo, o acesso e/ou a sua proliferação. Ainda assim, quando as medidas de prevenção adotadas não forem eficazes, o controle químico deve ser empregado e executado por empresa especializada com produtos desinfetantes regularizados pelo Ministério da Saúde, conforme descrito na resolução RDC ํ․ 216/04 e seguido na maioria dos restaurantes da região central (BRASIL, 2004).

Em relação ao grupo de itens relacionados ao abastecimento de água, pode-se observar que, nas duas regiões estudadas, esse grupo obteve destaque, principalmente na região sul onde os itens desse grupo apresentaram maior conformidade com as Boas Práticas quando comparados aos da região central.

De acordo com a Organização das Nações Unidas (UNITED NATIONS, 2010), cerca de 80\% de todas as doenças que acometem os países em desenvolvimento provêm de água de má qualidade; portanto, a resolução RDC n. 216/04 (BRASIL,2004) estabelece que as áreas de abastecimento de água devem conter reservatório edificado e/ou revestido de materiais que não comprometam a qualidade da água, livre de rachaduras, vazamentos, infiltrações e descascamentos, em adequado estado de higiene e conservação, devidamente tampado e higienizado, em um intervalo máximo de seis meses, devendo serem mantidos registros da operação de higienização. Deve ser utilizada somente água potável para manipulação dos alimentos e, quando utilizada solução alternativa, a potabilidade deve ser testada semestralmente mediante laudos laboratoriais.

Os itens relacionados à exposição ao consumo do alimento preparado nos restaurantes da região sul apresentaram maior média de conformidade com as Boas Práticas, enquanto os restaurantes da região central apresentaram a menor média de conformidade (11,4\%), como observado na Tabela 2. De acordo com a metodologia utilizada para avaliação das Boas Práticas, os itens do grupo que justificam as inadequações dos restaurantes da região central estão relacionados ao monitoramento da temperatura dos equipamentos que expõe os alimentos e à presença de barreiras de proteção que previnam a contaminação dos alimentos pelos consumidores 
ou outras fontes.

No estudo de Zandonadi et al. (2007), foi demonstrado que, em um sistema de distribuição centralizado, existe a probabilidade de contaminação dos alimentos pelos consumidores, uma vez que estes mantêm contato direto com os alimentos expostos no balcão de distribuição. A fonte de contaminação pelos consumidores se dá com a simples atitude de mexer nos cabelos perto das preparações, seja pelo contato direto do cabelo que cai sobre a preparação, seja por sua transmissão ao ambiente. Assim também, como na boca há diversos tipos de microrganismos, a atitude de tossir, cantar ou falar sobre os alimentos é tão contaminante quanto espirrar. Os microrganismos provenientes das gotículas de saliva expelidas, quando alguma dessas atitudes ocorre, podem depositar-se sobre os alimentos, contaminando-os. A roupa, as partes do corpo e os demais objetos são igualmente veiculadores de microrganismos aos alimentos.

Além disso, quando o fluxo de consumidores diminui, as preparações ficam expostas por um tempo maior, possivelmente em temperatura favorável ao desenvolvimento microbiano, o que favorece a contaminação (QUEIROZ et al., 2000). Sendo assim, é necessário que os consumidores sejam conscientizados sobre as atitudes apropriadas durante a montagem de seus pratos, a fim de serem evitadas não só possíveis contaminações como também as doenças transmitidas por alimentos contaminados.

Na região sul, foi verificado que os itens referentes à responsabilidade técnica apresentaram menor média de conformidade (25\%). Esses restaurantes não possuíam responsável devidamente capacitado e habilitado para desenvolver as atividades específicas necessárias às BPs. O Conselho Regional de Nutrição estabelece que o nutricionista é o profissional habilitado para assumir as atividades de planejamento, coordenação, direção, supervisão e avaliação na área de alimentação e nutrição; portanto, é o profissional que possui habilidades técnicas para assumir a responsabilidade de estabelecimentos produtores de alimentos. No entanto, a resolução RDC 216/04 (BRASIL,2004) descreve que o responsável pela manipulação dos alimentos deve ser o proprietário ou funcionário designado, devidamente capacitado, e que o responsável pelas atividades de manipulação dos alimentos deve ser, comprovadamente, submetido a curso de capacitação, abordando, no mínimo, os temas relacionados à contaminação alimentar, doenças transmitidas por alimentos, manipulação higiênica dos alimentos e Boas Práticas.

Observou-se que, na região central, dentro do grupo edificação, instalações, equipamentos, móveis e utensílios, os itens que apresentaram maior número de inadequações foram os relacionados às luminárias localizadas na área de preparação, pois não estavam protegidas contra explosão e/ou quedas acidentais, a não existência de lavatório exclusivo para higiene das mãos na área de manipulação, à falta de sabonete líquido inodoro antisséptico, toalhas de papel não reciclado e/ou outro sistema higiênico e seguro de secagem das mãos. É possível verificar que essas inadequações estão relacionadas, pois a falta de sabonete inodoro antisséptico e/ou toalhas de papel não reciclado são devido à inexistência de lavatório exclusivo para higiene das mãos.

Dados semelhantes foram encontrados por Da Veiga, Doro e Bombo (2006), que, em seu estudo, observaram que os estabelecimentos de comercialização de alimentos não possuíam lavatórios exclusivos para lavagens de mãos, dentro das áreas de manipulação dos alimentos, 
aumentando o risco de contaminação também durante o preparo das refeições. Na região sul, o maior número de inadequações, dentro desse grupo, foi devido ao dimensionamento das edificações que não eram compatíveis com todas as operações e à inexistência de separação entre diferentes atividades por meios físicos ou por outros meios eficazes. Segundo a resolução RDC 216/04 (BRASIL, 2004), tal separação das atividades é importante para evitar a contaminação cruzada entre gêneros.

No grupo higienização das instalações, equipamentos, móveis e utensílios, apresentaram, na região central, maior número de inadequações, bem como os itens relacionados ao registro de higienização, que não são realizados de rotina e os relacionados à utilização de uniformes apropriados. Segundo a resolução RDC 216/04 (BRASIL, 2004), estes devem ser diferenciados daqueles utilizados na manipulação de alimentos pelos funcionários responsáveis pela higienização das instalações sanitárias. Esse dado é semelhante ao encontrado na região sul, uma vez que, na metade dos restaurantes avaliados, os funcionários designados à higienização das instalações sanitárias não apresentavam uniformes diferenciados dos uniformes usados pelos manipuladores. Além disso, os produtos saneantes não eram identificados e guardados em local reservado para essa finalidade. Esse fato está, possivelmente, relacionado com o dimensionamento do local, conforme descrito no grupo edificação, instalações, equipamentos, móveis e utensílios.

O item do grupo manejo dos resíduos que apresentou maior número de inadequação nos restaurantes da região central foi o de coletores de lixo das áreas de preparação e armazenamento que devem ser dotados de tampas acionadas sem contato manual. Em alguns estabelecimentos, os coletores de lixo apresentavam tampas, porém não possuíam pedais, o que pode contribuir para a contaminação entre manipulador e alimento. Na região sul, esse grupo não apresentou inadequações importantes.

Os itens com maior número de inadequações do grupo manipuladores, na região central e na região sul, foram representados pela falta de cartazes com orientações sobre a correta antissepsia das mãos e demais hábitos de higiene, em locais de fácil visualização, inclusive nas instalações sanitárias e lavatórios. Esse fato, possivelmente, está relacionado, na região central, com a ausência dos produtos para higiene das mãos e à falta do lavatório exclusivo para higienização das mãos na área de manipulação; e, na região sul, devido ao dimensionamento das instalações, conforme descrito no grupo edificação, instalações, equipamentos, móveis e utensílios. Além dessa inadequação, na região central foi observado que os manipuladores, durante a produção das refeições, realizavam algumas atitudes que poderiam contribuir para a contaminação das preparações, como falar desnecessariamente, cantar, assoviar, tossir ou realizar outros atos que poderiam contaminar os alimentos. Esse dado é semelhante ao encontrado no estudo de Oliveira, Brasil e Taddei (2008), no qual se evidenciaram práticas sanitárias inadequadas em relação aos manipuladores, que falavam, cantavam, tossiam, espirravam durante a manipulação do alimento e não realizavam, corretamente, a técnica de lavagem das mãos. A adoção de técnicas corretas de manipulação dos alimentos e a conscientização dos profissionais envolvidos são fundamentais como medida de controle das infecções de origem alimentar, a detecção e a rápida correção das falhas no processamento dos alimentos, bem como a adoção de medidas preventivas, são as principais estratégias para o controle de qualidade dos alimentos (GÓES et al., 2001).

O grupo relacionado à documentação e registro apresentou, na região central e na região sul, 
o grupo com maior número de itens de não conformidade com as Boas Práticas. Esse resultado é semelhante ao encontrado no relatório do Programa Paulista de Análise Fiscal de Alimentos, biênio 2005-2006, no qual o item documentação e registros apresentou o maior índice de não conformidade para todos os ramos de atividades inspecionados. Esse item contempla o Manual de Boas Práticas e as descrições dos procedimentos Operacionais Padronizados (POP), que estabelecem instruções para realização de operações rotineiras e específicas da produção, armazenamento e do transporte de alimentos (SÃO PAULO, 2007). No trabalho realizado por Yamamoto et al. (2004), foi verificado que o Manual de Boas Práticas (MBP) não existia na maior parte dos estabelecimentos pesquisados e, quando existia, na grande maioria das vezes, ficava em poder do proprietário ou gerente, impedindo o acesso ao documento pelos demais funcionários. Segundo White et al. (2005), a elaboração do Manual de Boas Práticas é fundamental para se alcançar manipulação adequada de acordo com os critérios higiênicos recomendados.

Os resultados encontrados nos grupos matérias-primas, ingredientes e embalagens, preparação do alimento, armazenamento e transporte do alimento preparado não apresentaram itens de inadequação em quantidade importante, uma vez que esses dados foram dispersos no grupo.

Os dados da Tabela 3 estão representados pela relação entre os grupos de adequação e preço da refeição oferecida nos restaurantes da região central e da região sul da cidade de São Paulo, SP.

Tabela 3 - Distribuição da relação entre os grupos de adequação e preço da refeição oferecida nos restaurantes da região central e da região sul de São Paulo (SP). São Paulo, 2012

\begin{tabular}{|c|c|c|c|c|c|c|c|c|}
\hline \multirow[t]{2}{*}{$\begin{array}{c}\text { Grupos de } \\
\text { adequação (\%) }\end{array}$} & \multicolumn{2}{|c|}{$\begin{array}{c}\text { Preço } \\
R \$<20,00 \\
\text { Região Central }\end{array}$} & \multicolumn{2}{|c|}{$\begin{array}{c}\text { Preço } \\
\mathrm{R} \$>20,00 \\
\text { Região Central }\end{array}$} & \multicolumn{2}{|c|}{$\begin{array}{c}\text { Preço } \\
\mathrm{R} \$<20,00 \\
\text { Região Sul }\end{array}$} & \multicolumn{2}{|c|}{$\begin{array}{c}\text { Preço } \\
\mathbf{R} \$>20,00 \\
\text { Região Sul }\end{array}$} \\
\hline & $\mathrm{N}$ & $\%$ & $\mathrm{~N}$ & $\%$ & $\mathrm{~N}$ & $\%$ & $\mathrm{~N}$ & $\%$ \\
\hline Grupo 1 - 76 a 100 & 0 & 0 & 5 & 31,2 & 0 & 0 & 8 & 50,0 \\
\hline Grupo 2 - 51 a 75 & 6 & 37,5 & 3 & 18,7 & 0 & 0 & 7 & 43,7 \\
\hline Grupo 3 - 0 a 50 & 2 & 12,5 & 0 & 0 & 1 & 6,2 & 0 & 0 \\
\hline Total & 8 & 50,0 & 8 & 49,9 & 1 & 6,2 & 15 & 93,7 \\
\hline Teste exato de Fishe & \multicolumn{4}{|c|}{$p=0,026$} & \multicolumn{4}{|c|}{$p=0,062$} \\
\hline
\end{tabular}

teste exato de Fisher no total $=0,005$

De acordo com os dados obtidos nesta pesquisa, foi possível verificar que, apesar do tamanho da amostra, os resultados sugerem que os estabelecimentos com preços mais elevados condicionam uma melhor qualidade dos serviços em relação às Boas Práticas.

Os resultados satisfatórios encontrados nas duas regiões podem ser avaliados como decorrência de uma limitação deste estudo, uma vez que, possivelmente, os restaurantes que estivessem em condições muito inadequadas não tenham aceitado participar da pesquisa. 
Observa-se, na Tabela 3, que houve significância em relação à porcentagem de adequação e preço da refeição na Região Central. Na Região Sul, essa condição não foi observada, possivelmente por serem restaurantes mais novos.

\section{CONCLUSÃO}

De acordo com os resultados obtidos, é possível concluir que a maior parte dos restaurantes avaliados da região central e da região sul da cidade de São Paulo (SP), de um modo geral, tiveram um bom desempenho em relação às conformidades com as Boas Práticas e que, na Região Central, os restaurantes que apresentaram custo mais elevado das refeições tiveram maior adequação à legislação sanitária vigente em nível federal.

\section{REFERÊNCIAS}

AKUTSU, R. C. et al. Adequação das boas práticas de fabricação em serviços de alimentação. Revista de Nutrição, v. 18, n. 3, pp. 419-427, 2005.

ANVISA. Agência Nacional de Vigilância Sanitária. Gerência Geral de Alimentos. Cartilha sobre Boas Práticas para Serviços de Alimentação. Brasília: Ministério da Saúde, 2005.

ARAÚJO, W. M. C. Qualidade dos alimentos comercializados no Distrito Federal no período de 1997-2001. 2002. 79f. Dissertação (Mestrado em Nutrição Humana) Universidade de Brasília, Distrito Federal, 2002.

AVEGLIANO, R. P. et al. Check List unificado para classificação higiênico-sanitária de restaurantes. Higiene Alimentar, São Paulo, v. 24, n. 183, pp. 45-56, 2010.

BRAITE DE OLIVEIRA, M. E. Surtos de Doenças Transmitidas por Alimentos no estado de São Paulo, 2008-2010. 2013. Dissertação (Mestrado em Saúde Coletiva) - Faculdade de Ciências Médicas da Santa Casa de São Paulo, São Paulo. 2013.

BRASIL. Ministério da Saúde. Secretaria de Vigilância em Saúde. Portaria no. 1.428, de 26 de novembro de 1993. Aprova o Regulamento Técnico para Inspeção Sanitária de Alimentos, as Diretrizes para o Estabelecimento de Boas Práticas de Produção e de Prestação de Serviços na Área de Alimentos. Brasília, DF, 1993.

. Portaria SVS n. 326, de 30 de julho de 1997. Aprovar o Regulamento Técnico: Condições

Higiênico-Sanitárias e de Boas práticas de Fabricação para Estabelecimentos Produtores/Industrializadores de Alimentos. Brasília, DF, 1997.

Resolução - RDC no. 275, de 21 outubro de 2002. Dispõe sobre o Regulamento Técnico de Procedimentos Operacionais Padronizados aplicados aos Estabelecimentos Produtores/Industrializadores de Alimentos e a Lista de Verificação das Boas Práticas de Fabricação. Brasília, DF, 2002. 
. Ministério da Saúde. Portaria no 216/Brasília, de 15 de setembro de 2004. Regulamento Técnico sobre Condições Higiênico-Sanitárias e de Boas Práticas para Serviços de Alimentação. Diário Oficial da União, 2004.

Ministério da Saúde. Secretaria de Vigilância em Saúde. Morbidade e mortalidade por doenças transmissíveis no Brasil. In. Uma análise da situação da saúde e da agenda nacional e internacional de prioridades em saúde. Brasília, DF: Ministério da Saúde, 2009. 38p. Disponível

em: $<$ http://portalsaude.saude.gov.br/portalsaude/arquivos/pdf/2013/Fev/28/saudebrasil2009 parte1 c ap3.pdf>. Acesso em: 08 ago. 2013.

CASTRO, M. F. M.; SCHIMIDT, F. L.; LEITÃO, M. F. F Boas Práticas agrícolas e aplicação do sistema APPCC no cultivo e processamento industrial do café obtido pela via seca. Higiene Alimentar, v. 16, n. 96, pp. 39-42, 2002.

CODEX ALIMENTARIUS. Food Hygiene basic texts. 2. ed. Rome: FAO; WHO; 2001.

DA VEIGA, C. F.; DORO, D. L.; BOMBO, D. L. Estudo das condições sanitárias dos estabelecimentos comerciais de manipulação de alimentos do município de Maringá. Higiene Alimentar, v. 20, n.138, pp. 28-36, jan./fev., 2006.

FORSYTHE, S. J. Microbiologia da segurança alimentar. Porto Alegre: Artmed, 2000, 424p.

GÓES J. A. W. et al. Capacitação dos manipuladores de alimentos e a qualidade da alimentação servida. Higiene Alimentar, v. 15, n. 82, pp. 20-22, 2001.

MÜRMANN, L.; MALLMANN, C. A.; DILKIN, P. Temperaturas de armazenamento de alimentos em estabelecimentos comerciais na cidade de Santa Maria, RS. Acta Scientiae Veterinariae, v. 33, pp. 309-313, 2005.

HOBBS, B. C.; ROBERTS, D. Toxinfecções e controle higiênico-sanitário de alimentos. São Paulo: Varela, 1999. 376 p.

OLIVEIRA, M. N.; BRASIL, A. L. D.; TADDEI, J. A. A. C. Avaliação das condições higiênicosanitárias das cozinhas de creches públicas e filantrópicas. Ciência \& Saúde Coletiva, v. 13, n. 3, pp. 1051-1060, 2008.

UNITED NATIONS. United Nations High Commissioner for Human Rights. World Health Organization. The Right to Water. Geneva: United Nations, 2010 (Fact sheet, n. 35). Disponível em: <http://www.ohchr.org/Documents/Publications/FactSheet35en.pdf>. Acesso em: 13 maio 2014.

QUEIROZ, A. T. A. et al. Boas práticas de fabricação em restaurantes "self-service" a quilo. Higiene Alimentar, v. 14, n. 78, pp. 48-49, 2000.

SACCOL, A. L. F. et al. Lista de Avaliação para Boas Práticas em Serviços de Alimentação: RDC216. São Paulo: Varela, 2006. 47p. 
SACCOL, A. L. F. et al. Hygiene and sanitary conditions in self-service restaurants in São Paulo, Brazil. Food Control, v. 33, n. 1, p. 301-305, 2013.

SÃO PAULO. Secretaria de Estado da Saúde. Centro de Vigilância Sanitária. Relatório Final Programa Paulista de Análise Fiscal de Alimentos: Biênio 2005-2006. São Paulo, 2007. Disponível em: <http://200.144.0.250/download/rel final pp0506.pdf>. Acesso em: 08 maio 2012.

TOMICH, R. G. O. et al. Metodologia para avaliação das boas práticas de fabricação em indústrias de pão de queijo. Ciência e Tecnologia de Alimentos, Campinas, v. 25, n. 1, mar. 2005. Disponível em: $\quad<$ http://www.scielo.br/scielo.php?script=sci arttext\&pid=S010120612005000100019\&lng=en\&nrm=iso>. Acesso em: $13 \quad$ May 2014. http://dx.doi.org/10.1590/S0101-20612005000100019.

WEINGOLD, S. E.; GUZEWICH, J.; FUDALA, J. K. Use of Foodborne disease data for HACCP risk assessment. Journal of Food Protection, v. 57, p. 820-830, 1994.

WHITE, H. J. et al. Análise microbiológica das mãos dos manipulador e envolvidos no preparo de dietas enterais do Hospital Escola de Itajubá, MG. Nutrição em pauta, n. 70, pp. 46-49, 2005.

YAMAMOTO, D. C. et al. Caracterização das condições higiênico - sanitárias dos restaurantes "fastfood" de dois "shopping centers", em diferentes regiões do Município de São Paulo. Higiene Alimentar, v. 18, n. 122, pp. 14-20, 2004.

ZANDONADI, R. P. et al. Atitudes de risco do consumidor em restaurantes de autosserviço. Revista de Nutrição, v. 20, n. 1, pp. 19-26, 2007. 